\title{
SURPRISING BEHAVIOUR AND SINGULARITY IN THE SAINT VENANT APPROXIMATION FOR A FLUID
}

\author{
PAOLO LUCHINI (*)
}

\begin{abstract}
SunTO. - Si rassegna una linea di ricerca che, nell'arco di qualche anno, ha portato ad un sostanziale cambio di prospettiva riguardo ai modelli semplificati che permettono la descrizione delle correnti fluide quasi-unidimensionali, delle loro instabilità, e dei loro effetti su letti sabbiosi. Anche quando il flusso è considerato laminare, l'equazione di Saint-Venant del flusso quasi-unidimensionale può essere formulata in piú di un modo; si mostrerà che solo una di queste scelte è consistente con le equazioni tridimensionali complete di Navier-Stokes. Quando il flusso è turbolento la necessità di un modello di turbolenza, di solito quasi sempre del tipo della eddy viscosity, costituisce una complicazione addizionale; si mostrerà che un tale modello può cadere in forte contrasto con una simulazione numerica diretta dello stesso fenomeno, fino al punto di produrre risultati di segno opposto. Per di piú, la simulazione numerica completa del flusso su di un fondale ondulato esibisce un approccio non-monotono al suo limite di onde lunghe (quasi-unidimensionale), con la presenza di una sorprendente risonanza che non ha alcun analogo nel caso laminare e che dovrà essere l'oggetto di future ricerche.
\end{abstract}

$* * *$

ABSTRACT. - A research line is reviewed which, over a few years, led to a substantial change of perspective about the simplified models that underlie the description of quasi-onedimensional streams, their instabilities, and their effects upon sandy beds. Even when the flow is assumed to be laminar, the Saint-Venant equation of quasi-onedimensional fluid flow can be formulated in more than one manner; it will be shown that only one of these choices is consistent with the complete three-dimensional NavierStokes equations. When the flow is turbulent, an added complication is the presence of a turbulence model, most often of the eddy-viscosity type; it will be shown that such a model can be in strong contrast with a direct numerical simulation of the same phenomenon, even to the point of producing results of opposite sign. In addition, the complete numerical simulation of flow past an undulated bottom exhibits a non-monotonic approach to its long-wave, quasi-onedimensional limit, with a surprising resonance that has no laminar counterpart and must become the subject of future investigations.

(*) Presidente AIMETA, Università di Salerno - DIIN, Fisciano (SA), Italia. E-mail: luchini@unisa.it 


\section{INTRODUCTION}

When the scale of length over which a fluid flow evolves is much larger than the depth of the channel or duct that contains it, this scale contrast suggests a family of approximations where the flow is treated as a small perturbation away from the one that would occur in an infinitely long straight channel. Such approximations are widespread and commonly used for both environmental and industrial flow problems. Nonetheless, over several years in a joint research effort with the Institut de Mècanique des Fluides de Toulouse and other institutions, we have become aware that the slowly-varying (long-wave) limit of a channel flow is more complicated than one could a priori imagine, and hides several surprises. Some of these surprises have been uncovered in our published papers and will be reviewed in this presentation.

Whereas the largest difficulties arise in turbulent flow, even in laminar flow the long-wave formulation lends itself to non-unique interpretations, and needs a careful analysis in order to be formulated properly. We shall therefore begin by disambiguating the SaintVenant formulation for laminar flow in a manner which was established in 2010 [1].

\section{Consistent SAint-VEnAnt approximation}

The analysis of slowly-varying channel flow, from (laminar) films to (turbulent) oceans, is often performed with depth-averaged equations of motion. In inviscid flow these are named the "shallow-water" equations

$$
\begin{aligned}
& h_{t}+(U h)_{x}=0 \\
& U_{t}+U U_{x}+p_{x} / \rho=0
\end{aligned}
$$

with $b$ representing the water height in the channel, $U$ its mean (depthaveraged) velocity and $p, \rho$ pressure and density. In viscous, laminar or turbulent, flow a similar depth averaging produces the Saint-Venant equations,

$$
\begin{aligned}
& h_{t}+(U h)_{x}=0 \\
& U_{t}+U U_{x}+p_{x} / \rho=g \sin \alpha-\tau_{w} / \rho h
\end{aligned}
$$


with $g \sin \alpha$ being the longitudinal component of gravity along a possibly inclined duct with slope angle $\alpha$, and wall shear stress $\tau_{w}$ usually accounted for through a friction coefficient.

These equations are widely used for environmental problems in the atmosphere and the ocean, as well as in rivers [2, 3], and successfully describe the propagation of flood waves, Rossby or Kelvin waves. They also describe gravity currents and snow avalanches [4], as well as granular flow along slopes $[5,6]$. They are also a useful model for engineering calculations, e.g. the pressure drop and phase distribution of twophase flow in ducts $[7,8]$. Many examples arise in hydrodynamic stability theory, such as the roll-wave instability $[9,10,11,12]$ or the stability of an erodible bed $[13,14,15,16]$.

Nevertheless there is trouble around the corner. Whereas eqs.(1) are consistent with a uniform velocity profile, the Saint-Venant equations (2) stem from averaging a velocity profile that becomes zero at the wall, and to be consistent require a correction coefficient $\beta$ accounting for the integral of this non-uniform velocity profile. Equation (2b) thus becomes

$U_{t}+\beta U U_{x}+p_{x} / \rho=g \sin \alpha-\tau_{w} / \rho h$

One difficulty with eq.(3) is that more than one choice is possible for both the correction coefficient $\beta$ and the expression of the shear stress $\tau_{w}$. In plane-channel flow, $\beta$ can be chosen as

$$
\beta= \begin{cases}6 / 5 & \text { from the integrated momentum equation } \\ 54 / 35 & \text { from the integrated energy equation } \\ 1 & \text { when velocity corrections are considered } \\ & \text { negligible in turbulent flow. }\end{cases}
$$

Another difficulty arises in connection with the wall shear stress $\tau_{w}$, most often expressed as

$$
\frac{\tau_{w}}{\rho}=c_{f} \frac{U^{2}}{2}
$$

where, for laminar flow $c_{f}=64 / \mathrm{Re}$, and various expressions have been proposed for turbulent flow. The problem here is that this assumed local relationship between shear stress and velocity is instantaneous (takes effect immediately, with no phase shift when a sinusoidal pertur- 
bation is applied), in contrast with the essential role that a phase lead between stress and velocity plays in the short-wave instability theory of Benjamin [17].

An answer to these indeterminacies was given for laminar flow in [1] and for turbulent flow in [18]. Following [1], the asymptotic analysis of a channel with slowly varying height $h(x, t)$ involves a two-scale expansion such that $t=T / \varepsilon, x=X / \varepsilon$, and $T, X$ remain $\mathrm{O}(1)$ as $\varepsilon \rightarrow 0$. It follows that $h_{t}=\mathrm{O}(\varepsilon), h_{x}=\mathrm{O}(\varepsilon)$, even though $h(x, t)$ itself is not required to be small. An expansion of the laminar Navier- Stokes equations in powers of $\varepsilon$ then leads to the following hierarchy: Order 0

$$
\begin{gathered}
v^{(0)}=0 \\
\nu u_{y y}^{(0)}=P_{x}^{(0)} / \rho
\end{gathered}
$$

Order 1

$$
\begin{gathered}
u_{x}^{(0)}+v_{y}^{(1)}=0 ; \quad P_{y}^{(1)}=0 \\
\nu u_{y y}^{(1)}-P_{x}^{(1)} / \rho=u_{t}^{(0)}+u^{(0)} u_{x}^{(0)}+v^{(1)} u_{y}^{(0)}=u_{t}^{(0)}+u^{(0)} u_{x}^{(0)}-u_{y}^{(0)} \int_{0}^{y} u_{x}^{(0)} \mathrm{d} y
\end{gathered}
$$

with boundary conditions $u_{y}^{(1)}(0)=u^{(1)}(b)=v^{(1)}(0)=0$ and $v^{(1)}(b)=h_{t}$, which also implicitly determine the pressure gradient $P_{x}^{(0)}$. The order- 0 solution is the classical Poiseuille flow, but with centerline velocity $u_{M}$ and pressure gradient $P_{x}^{(0)}$ which are yet unknown functions of the "slow" coordinates T,X:

$$
\begin{gathered}
u^{(0)}=\left[1-(y / h)^{2}\right] u_{M}, \\
P_{x}^{(0)}=-2 \rho \nu u_{M} / h^{2} .
\end{gathered}
$$

Integrals of the order- 1 momentum and energy equations are:

$$
\begin{aligned}
& \frac{\partial}{\partial T} \int_{0}^{h} u^{(0)} \mathrm{d} y+\frac{\partial}{\partial X} \int_{0}^{h} u^{(0)^{2}} \mathrm{~d} y+h P_{x}^{(1)}=-\tau^{(1)}=\frac{1}{\operatorname{Re}} u_{y}^{(1)}(h), \\
& \frac{\partial}{\partial T} \int_{0}^{h} \frac{u^{(0)^{2}}}{2} \mathrm{~d} y+\frac{\partial}{\partial X} \int_{0}^{h} \frac{u^{(0)^{3}}}{2} \mathrm{~d} y+h U P_{x}^{(1)}=-f^{(1)}=-\frac{2}{\operatorname{Re}} \int_{0}^{h} u_{y}^{(0)} u_{y}^{(1)} \mathrm{d} y,
\end{aligned}
$$

with the quantities on the right-hand side representing - $\tau^{(1)}$ : first-order correction to the wall shear stress.

- $f^{1}$ : first-order correction to the energy dissipation (entropy production).

These first-order corrections are absent in the classical formula- 
tion of the (either momentum- or energy-based) Saint-Venant equation; their absence explains why these two formulations are inconsistent and in contrast with each other. On the other hand, if both $\tau^{(1)}$ and $f^{1)}$ are included, then the problem contains more unknowns than equations and its solution becomes undetermined.

A solution to this conundrum is provided by the, also classical, variational property of the entropy production (dissipation), which states that the order-0 Stokes flow (not only in a 2D channel, but also in a 3D geometry) minimizes such dissipation. At the minimum of a function its firstorder perturbations are zero, and in fact in the present problem it is not hard to verify that, for an arbitrary velocity perturbation $u(1)$,

$$
\begin{aligned}
\frac{\operatorname{Re}}{2} f^{(1)}=\int_{0}^{h} u_{y}^{(0)} u_{y}^{(1)} \mathrm{d} y=\left[u_{y}^{(0)} u^{(1)}\right]_{0}^{h} & -\int_{0}^{h} u_{y y}^{(0)} u^{(1)} \mathrm{d} y= \\
& =u_{y}^{(0)}(h) u^{(1)}(h)-\operatorname{Re} P_{x}^{(0)} \int_{0}^{h} u^{(1)} \mathrm{d} y=0 .
\end{aligned}
$$

Therefore in the second of eqs.(4) the right-hand side can safely be replaced by zero. It follows that of the two classical formulations, the momentum equation (4a) and the energy equation (4b), only the energy form is consistent in the absence of a right-hand side. Luchini \& Charru [1] thus arrived at the following conclusions:

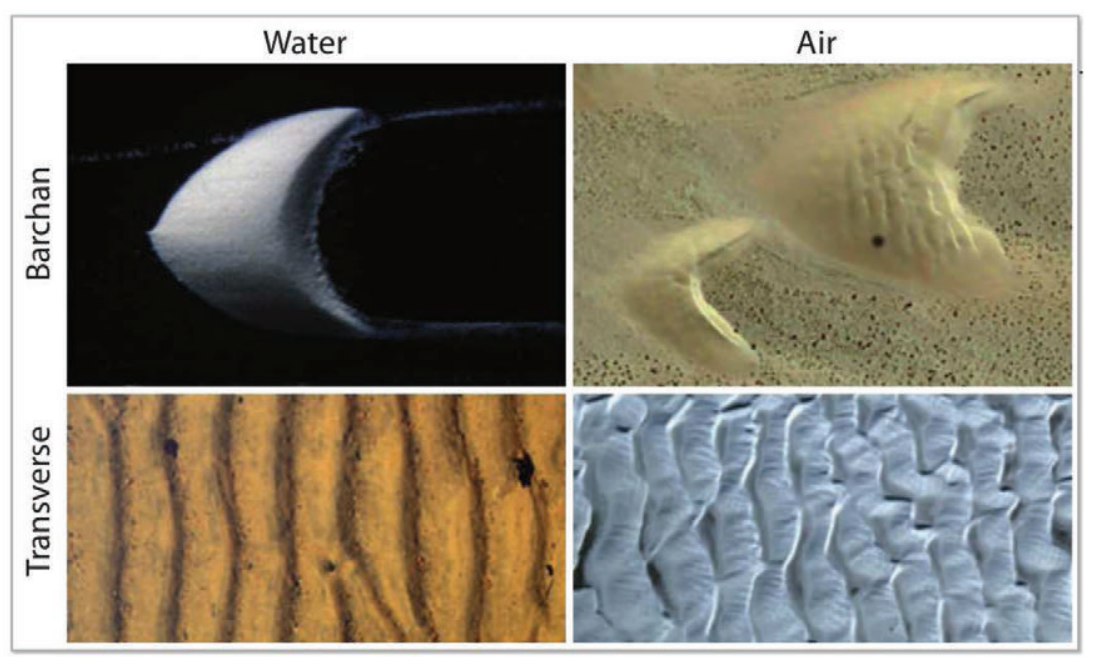

Fig. 1. A classical problem: the formation of ripples and dunes (from [21]). 
1. The energy form of the laminar Saint-Venant equation is the one that provides consistent correction coefficients:

$(6 / 5)\left(b U^{2} / 2\right)_{t}+(54 / 35)\left(b U^{3} / 2\right)_{x}+b U P_{x} / \rho=-3 v U^{2} / h$.

2. The Saint-Venant momentum equation needs to contain the wallshear stress as an unknown, and can actually be used to find out this shear stress after applying the energy equation:

$-\tau_{w}=\rho(b U)_{t}+(6 / 5) \rho\left(b U^{2}\right)_{x}+b P_{x}$.

3. For steady plane-channel flow, the long-wave wall shear stress is consequently given by

$$
\tau_{w}=\tau_{w}{ }^{(0)}\left[1-(4 \operatorname{Re} / 35) h_{x}\right],
$$

and it exhibits the expected phase lead.

Equation (8) reproduces the much earlier result, derived by laboriously solving the first-order equations for the corresponding velocity profile, of Benjamin [17] and Yih [19]. The just described energy method of [1] skips the calculation of the velocity profile, and thus generalizes with hardly any difficulty to $3 \mathrm{D}$ geometries and, as will be seen in $\$ 4$, to turbulent flow. It also clarifies that the role of the wall shear stress in the momentum Saint-Venant equation (2b) is not that of a parameter to be determined from an external costitutive equation, but quite on the contrary an unknown determined by eq.(2b) itself.

\section{EFFECTS OF THE PHASE LEAD UPON AN ERODIBLE BOTTOM}

An accurate model of quasi-onedimensional flow is necessary, among other purposes, in the context of an even longer-standing study of the formation of ripples and dunes $[20,21]$ under a stream of either water or air. Fig. 1 shows several examples. In the words of [21], the steady or oscillatory motion of a liquid above a granular bed leads to the formation of ripples. The sand ripples one observes on a beach at low tide are an example: these ripples were formed by the oscillations induced by the surface waves when the beach was covered with shallow 
water. The mechanism of their formation, related to fluid inertia, sits in the phase of bottom shear stress oscillations relative to oscillations of the bottom itself, with positive phase advance of the shear stress dragging the particles toward crests and negative phase delay towards troughs. The net particle flux toward crests can also be understood as the result of the mean steady drift flow (steady streaming). Similar structures are also observed on the continental shelf at water depths of 200-300 m, with a wavelength of the order of $1 \mathrm{~m}$.
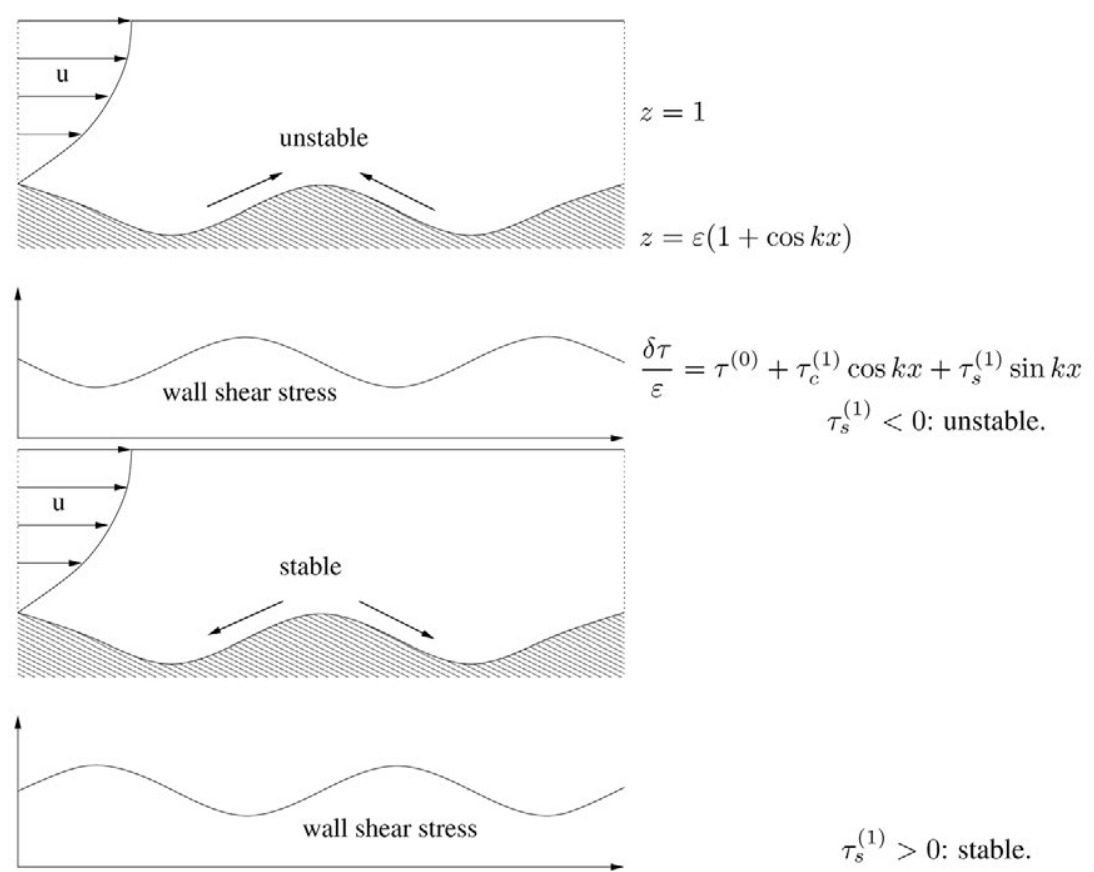

$$
\tau_{s}^{(1)}>0 \text { : stable. }
$$

Fig. 2. Whether a mound of sand casually formed over an erodible bed becomes higher (thus making the flat bottom unstable) or lower (thus tending to restore flatness) depends upon the phase of the channel depth perturbation relative to the shear perturbation it induces.

The classical explanation of the formation of ripples and dunes, within a linear-perturbation assumption, is illustrated in Fig. 2. As can be seen, a phase lag of the wall shear stress with respect to the deformation of the bottom (which translates into a modulated height of the channel) produces a larger shear on the downwards than on the 
upwards slope, and thus tends to drag sand particles down and smooth the shape. Under a phase lag the bottom tends to return flat and is stable. On the opposite, a phase lead implies that shear on the upwards slope is higher than shear on the downwards slope, and sand builds up on top of the pre-existing dune and makes it grow even more. In a linearized, small-perturbation setting both the bottom and shear perturbations appear as sinusoids. (This is actually a weakness of the theory, because experimentally observed dunes are frequently sharp-pointed and closer to a sawtooth then to a sinusoidal shape, but the necessary refinements will be left for future work.)

What needs to be emphasized in order to be compared with what follows is that the laminar theory of $\$ 2$, just as well as the older theories of $[17,19,22]$, does produce a negative quadrature component of stress (a phase lead), and therefore provides a justification for the unstable growth of dunes. The turbulent theories based on an eddy viscosity turbulence model, e.g. $[23,24]$, mimic the behaviour of their laminar counterparts and predict a qualitatively similar phase lead of the wall shear stress.

\section{Turbulent Saint-VEnant equations}

The extension of the consistent Saint-Venant formulation of $\$ 2$ to an eddy-viscosity model of turbulent flow was studied in [18]. Under turbulent-flow conditions, with a position-dependent eddy viscosity $v_{T}(y)$, the unperturbed velocity profile in a plane channel obeys

$$
\left[v_{T}(y) u_{y}^{(0)}\right]_{y}=\rho^{-1} P_{x}^{(0)} .
$$

This equation can be inverted to derive the eddy viscosity that is consistent with an arbitrary (modelled, measured or computed) velocity profile, thus giving

$$
\nu_{T}(y)=\frac{\tau^{(0)}}{\rho u_{y}^{(0)}}=\frac{y P_{x}^{(0)}}{\rho u_{y}^{(0)}}=\frac{-y \tau_{w}^{(0)}}{\rho h u_{y}^{(0)}} .
$$

The procedure to do so is well know, and was exploited in the past to deduce a turbulent-viscosity diagram from analytical [25], experimental [26] or numerical [27] mean-velocity data.

The simplest natural ansatz is to use the eddy viscosity derived 
from the mean flow to describe the first-order perturbation caused by a slow undulation of the channel bottom. This aim can be achieved in two ways:

- a perturbation expansion in the small undulation amplitude $\varepsilon$;

- our previously developed consistent form of the Saint-Venant equation.

The two yield coherent results. The order-1 velocity perturbation, in analogy with laminar flow, is obtained from a long-wave multiplescale expansion as

$$
\left(\nu_{T} u_{y}^{(1)}\right)_{y}-P_{x}^{(1)} / \rho=u^{(0)} u_{x}^{(0)}-u_{y}^{(0)} \int_{0}^{y} u_{x}^{(0)} \mathrm{d} y .
$$

A key property of eq.(9), consequent to its sharing one and the same $y$-dependent eddy viscosity with the mean flow, is that the corresponding energy equation is endowed with a minimum-dissipation property just as when viscosity is constant:

$$
f^{(1)}=\int_{0}^{h} \nu_{T}(y) u_{y}^{(0)} u_{y}^{(1)} \mathrm{d} y=0 .
$$

It follows that also in this turbulent case the energy form of the Saint-Venant equation is the consistent approach. As shown in [18], the energy equation can be generally formulated as

$$
\frac{\partial}{\partial t} \frac{\alpha h U^{2}}{2}+\frac{\partial}{\partial x} \frac{\beta h U^{3}}{2}+\frac{h U P_{x}}{\rho}=-f^{(0)}
$$

where

$$
\alpha=\frac{1}{h U^{2}} \int_{-h}^{0} u^{(0)^{2}} \mathrm{~d} y, \quad \beta=\frac{1}{h U^{3}} \int_{-h}^{0} u^{(0)^{3}} \mathrm{~d} y, \quad f^{(0)}=\nu \int_{-h}^{0} u_{y}^{(0)^{2}} \mathrm{~d} y .
$$

This also includes the laminar case (6), where the velocity profile is parabolic and therefore the form factors and the dissipation function respectively evaluate to $\alpha=6 / 5, \beta=54 / 35$ and $f^{(0)}=3 v U^{2} / h$.

It also remains true that, after the pressure gradient $P_{x}$ has been determined from the energy equation, the averaged momentum equation can be used to provide the correct wall-shear stress $\tau_{w}$. The momentum Saint-Venant equation can generally be written as

$-\rho^{-1} \tau_{w}=(b U)_{t}+\left(\alpha b U^{2}\right)_{x}+h \rho^{-1} P_{x}$ 
where $\tau_{w}(x, t)$ is the fluid shear stress on the bottom surface and $\alpha$ the same form factor as defined above. Once again, this formula includes the laminar result (8) as a special case.

\subsection{Approximate velocity profile of channel flow}

A rough but very simple approximation of the turbulent velocity profile of channel flow is a logarithmic function all the way from the bottom at $y=-b$ to the free surface at $y=0$, namely

$u^{(0)}=u_{M}+\kappa^{-1} u_{\tau} \log (1+z)$

(with $z=y / h$ ).

where $u_{M}$ is, as before, the centreline velocity, $u_{\tau}=\sqrt{\tau_{w} / \rho}$, and $\kappa$ is the universal von Kármán's constant with a value close to 0.4 .

Within this simplifying assumption the mean velocity $U$ is then given by

$$
U=\frac{Q}{h}=u_{M}+\frac{u_{\tau}}{\kappa} \int_{-1}^{0} \log (1+z) \mathrm{d} z=u_{M}-\frac{u_{\tau}}{\kappa},
$$

and the form factors work out to be:

$$
\begin{aligned}
\alpha-1 & =\left(\frac{u_{\tau}}{\kappa U}\right)^{2} \int_{-1}^{0}[1+\log (1+z)]^{2} \mathrm{~d} z=\left(\frac{u_{\tau}}{\kappa U}\right)^{2} \\
\beta-1 & =3\left(\frac{u_{\tau}}{\kappa U}\right)^{2} \int_{-1}^{0}[1+\log (1+z)]^{2} \mathrm{~d} z+\left(\frac{u_{\tau}}{\kappa U}\right)^{3} \int_{-1}^{0}[1+\log (1+z)]^{3} \mathrm{~d} z \\
& =3\left(\frac{u_{\tau}}{\kappa U}\right)^{2}-2\left(\frac{u_{\tau}}{\kappa U}\right)^{3} .
\end{aligned}
$$

From combining the momentum and energy equations (10) and (11), the total wall-shear stress ensues as

$$
\tau_{w}=\tau_{w}^{(0)}+\tau_{w}^{(1)}=\left(1+\frac{1}{\kappa^{2}} \frac{h U_{t}}{U^{2}}+\frac{2}{\kappa^{2}} \frac{h U_{x}}{U}\right) c_{f} \frac{\rho U^{2}}{2} .
$$

Particularly for steady flow (where $b U=$ const.), eq.(12) reduces to

$$
\tau_{w}=\left(1-\frac{2}{\kappa^{2}} h_{x}\right) c_{f} \frac{\rho U^{2}}{2} \text {. }
$$

Equation (13) was the main result of Luchini \& Charru [18]. Within an eddy-viscosity model, in turbulent just as in laminar flow, on 
an undulated surface the first-order correction to wall shear stress undergoes a phase lead.

\section{QUESTIONING THE EDDY-VISCOSITY MODEL}

Whereas the analysis of [18] qualitatively confirmed, and quantitatively made more precise, the already mainstream theory that the long-wave limit of channel flow over an erodible bottom induces a phase lead, it also opened an opportunity for testing the eddy-viscosity model upon which this deduction is based. Equation (9) can be read as establishing an equivalence between the effect of a perturbation in the order- 0 velocity profile $u^{(0)}$ and a volume force:

$$
\left(\nu_{T} u_{y}^{(1)}\right)_{y}-P_{x}^{(1)} / \rho=\underbrace{u^{(0)} u_{x}^{(0)}-u_{y}^{(0)} \int_{0}^{y} u_{x}^{(0)} \mathrm{d} y}_{\text {equivalent volume force }} .
$$

The problem of flow in a straight channel with an added volume force is computationally much easier than flow in an undulated channel, and enables a rare comparison between its solution by timeresolved direct numerical simulation (DNS) and the prediction of an eddy-viscosity model.

Russo \& Luchini [28] applied DNS to the simplified problem depicted in Fig. 3, using the equivalence between the effect of a slowly varying depth and an effective volume force.

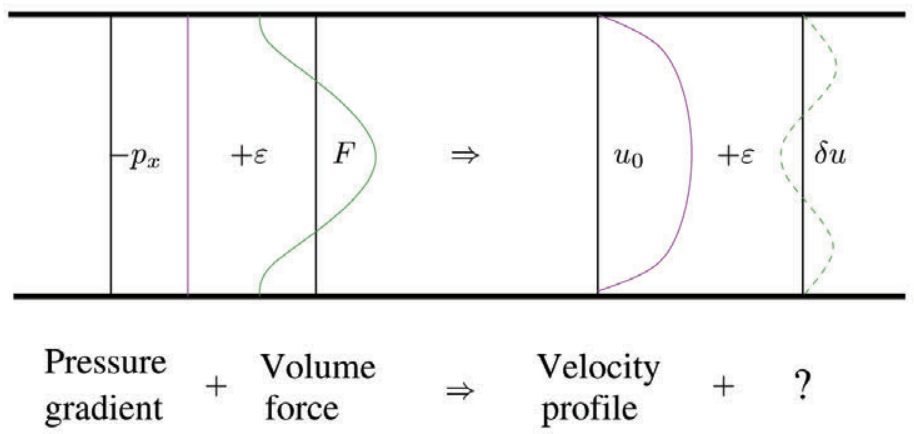

Fig. 3. The response of channel flow to a volume force was analysed through direct numerical simulation. Besides being relevant to the formation of dunes, this is a simple enough problem to afford a head-to-head comparison between eddy viscosity and DNS. 

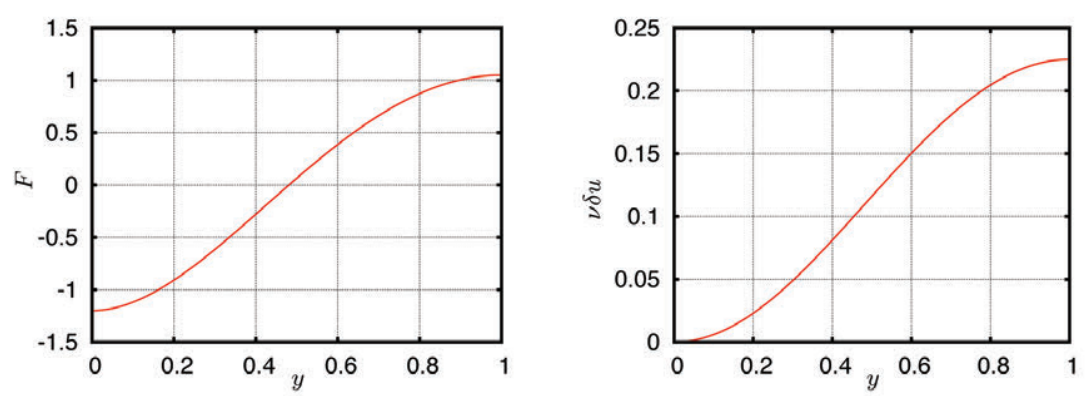

Fig. 4. Equivalent force to an undulated channel and its ensuing velocity perturbation for laminar flow.

\subsection{The example of laminar flow}

The volume force relevant to Luchini \& Charru's [1] laminar problem is

$$
F(y)=u_{0}^{2}(y)-\overline{u_{0}^{2}}=\left(3 y-\frac{3}{2} y^{2}\right)^{2}-\frac{6}{5}
$$

Solving $v \delta u_{y y}+F=0$ provides the velocity perturbation as

$$
v \delta u=\left(24 y^{2}-30 y^{4}+18 y^{5}-3 y^{6}\right) / 40
$$

Both $\delta u$ and $F$ are plotted in Fig. 4. The interesting observation here is that the total force (the integral of $F$ ) is set to zero, using the property that a constant can always be absorbed in $-p_{x}$; this is important because conservation of momentum reads $\tau_{w}=-p_{x}+\varepsilon \int_{0}^{1} F \mathrm{~d} y$, and therefore $\tau_{w}$ is unchanged with this choice. Yet, as shown in Fig. 5, even when the integral of force is zero the integral of the velocity perturbation is not. In fact $\delta u$ is everywhere positive, which implies that a net flow rate is generated with no net reaction force. This is somewhat unintuitive until one thinks carefully about it, but still it is generally true, and actually is a consideration also relevant to steady streaming $[29,30]$.

\subsection{Forced turbulent flow with an eddy-viscosity turbulence model}

For small perturbations to a parallel flow, most existing turbulence models reduce to a $y$-dependent eddy viscosity (convection of the turbulent energy is irrelevant in parallel flow). Among these, the least 
biased choice seems to be the eddy viscosity that is consistent with the unperturbed velocity profile, as described by [25, 26, 27, 18]. The only difference is that in the present section the mean velocity profile will be the discretized one obtained from DNS rather than its approximation adopted in $\$ 4$.
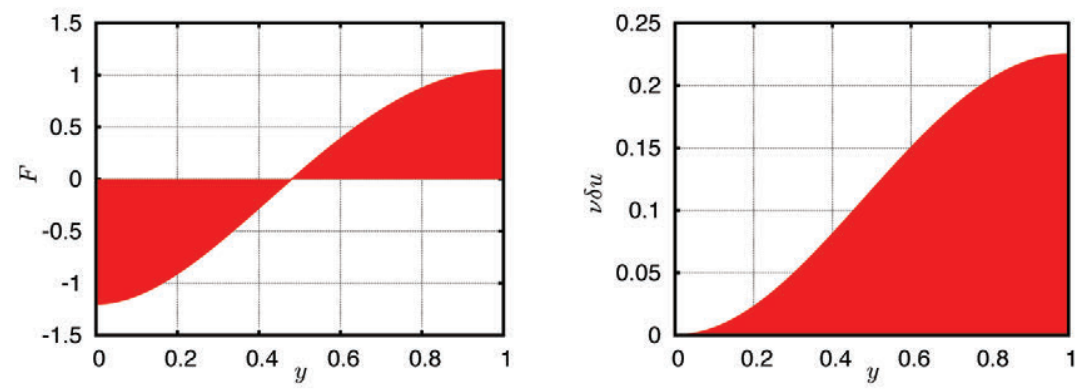

Fig. 5. Property of the laminar solution: a zero net force generates a non-zero flow rate.
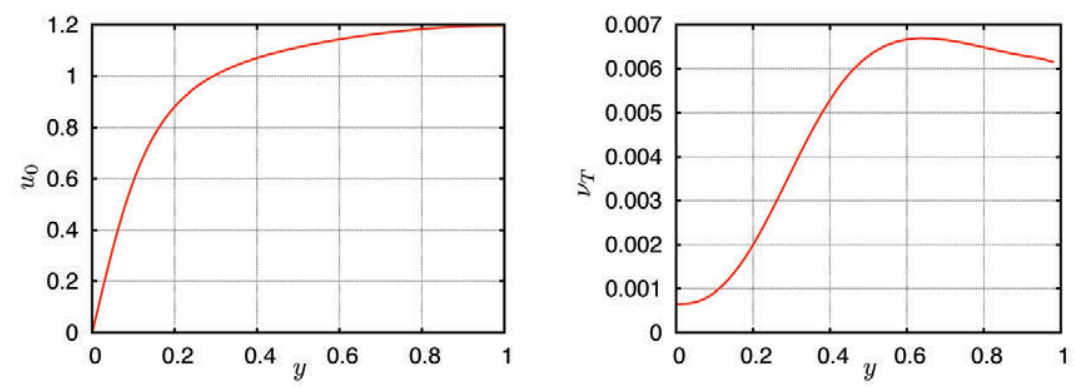

Fig. 6. Mean velocity profile from DNS at Re $=1700$ and eddy-viscosity profile deducted from it according to eq.(17).

Thus

$\nu_{T} \frac{\partial u_{0}}{\partial y}=\tau=(1-y) \tau_{w} \quad \Rightarrow \quad \nu_{T}(y)=\frac{(1-y) \tau_{w}}{\partial u_{0} / \partial y}$.

With $u_{0}$ from DNS data at $R e=1700$, the resulting $v_{T}$ is displayed in Fig. 6 .

Solving $\left(v_{T} \delta u_{y}\right)_{y}+F=0$ gives

$$
\delta u=-\int_{0}^{y} \nu_{T}^{-1}\left(\int_{0}^{y} F \mathrm{~d} y\right) \mathrm{d} y .
$$


With $F(y)=u_{0}^{2}(y)-\bar{u}_{0}^{2}$, where $u_{0}$ is the numerically obtained mean velocity, the corresponding result at $R e=1700$ is displayed in Fig. 7. We remark that once again $\delta u$ is everywhere positive: a zero net force generates a positive flow rate.

\subsection{Computed mean linear response of a forced turbulent flow}

Even though a turbulent flow is nonlinear, a mean linear response can be defined for small perturbations to the mean flow. This fairly intuitive concept is mathematically somewhat hard to frame precisely; nevertheless the mean linear response can be "measured", either in an experiment [31] or in a DNS [32], by imposing a volume force weighted by a small parameter $\varepsilon$, performing a finite difference over $\varepsilon$ and averaging over a long enough time. A balance has to be struck between linearity (improving with small $\varepsilon$ ) and statistical error associated to a finite averaging time (decreasing with large $\varepsilon$ ).
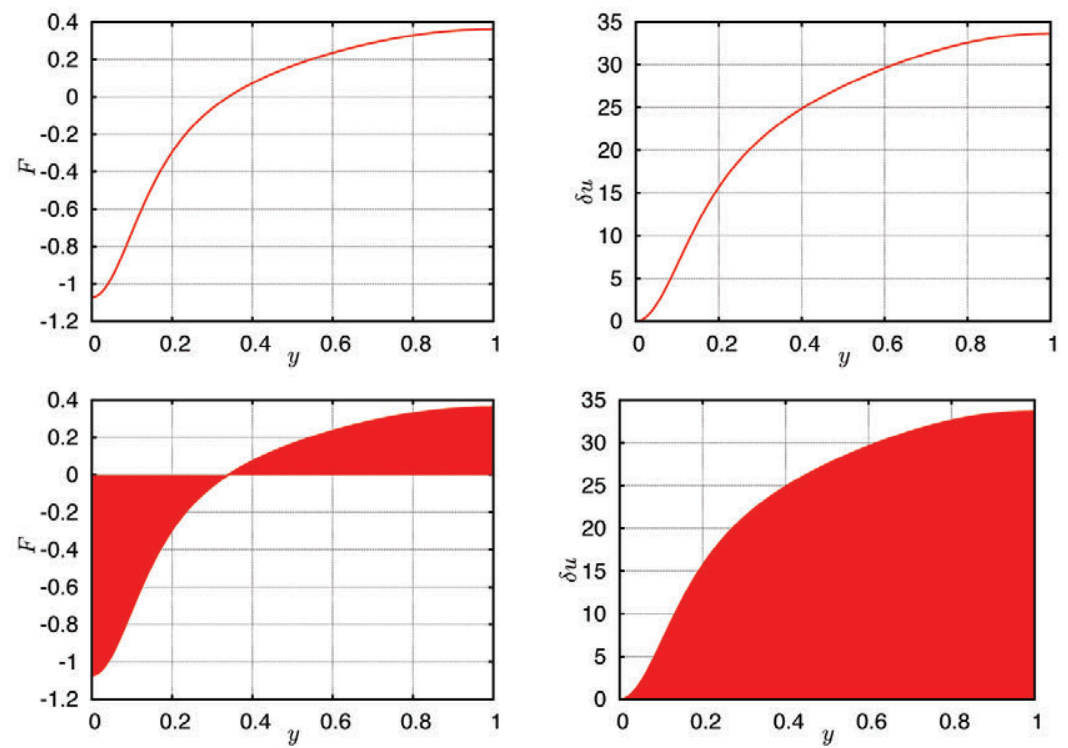

Fig. 7. Equivalent force and corresponding velocity perturbation as obtained from eddy viscosity.

In [28] we used the channel DNS code already developed for previous purposes in our laboratory [33], with a Fourier-Fourier-compact- 
difference discretization over a $4 \pi \times 2 \pi \times 2$ periodic box. The result was very surprising: as shown in Fig. 8 the velocity perturbation is not at all of one and the same sign, like the eddy-viscosity model had predicted, and in fact its integral, the flow-rate perturbation, is negative. A comparison between the numerical and eddy-viscosity velocity perturbations is exhibited in Fig. 9.

\subsection{Conclusions of Russo E Luchini [28]}

1. The linear response of a channel flow to a small volume force provides a valuable test bed for the comparison of DNS and turbulence models.

2. The DNS results are essentially different, and even of opposite sign, than the predictions of a commonplace eddy-viscosity model. Provably, no (positive) eddy viscosity can match the DNS.

3. Turbulence-convection models are not going to change this conclusion, since in a parallel flow convection has no consequence.

4. For applications such as flow along a wavy bottom, the quadrature component of wall shear stress turns out to be positive: in contrast to previous results, the erodible bottom is now predicted to be stable against long waves.

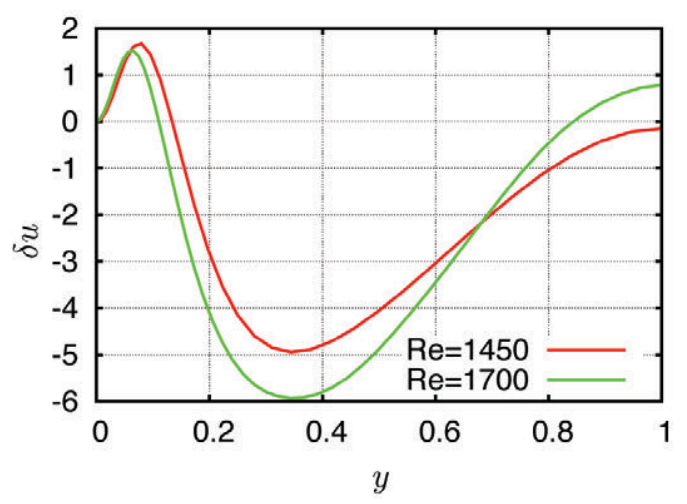

Fig. 8. Velocity response to a volume force from DNS.

These conclusions highlight a contradiction. Whereas in laminar flow the relative phase of shear stress with respect to the perturbation of the bottom leads to the deduction that an erodible bed is unstable at long wavelength, DNS of turbulent flow shows that deriving a similar conclusion from an eddy viscosity model is false. There were two implications: 
on one side eddy viscosity models are proved unreliable way beyond what might have been suspected; on the other hand a mystery remains about erodible beds, which in practice are indeed unstable. Trying to clarify this contradiction led to the development to be described next.

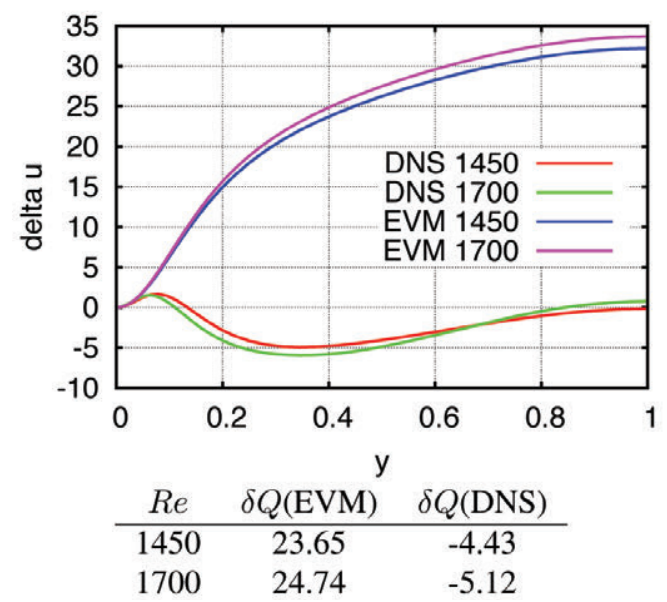

Fig. 9. Comparison between eddy viscosity and DNS. Until we know better, setting the flow-rate perturbation to zero is a better model than eddy viscosity.

\section{IMMERSED-BOUNDARY DIRECT NUMERICAL SIMULATION OF TURBULENT FLOW PAST A WAVY BOTTOM}

Whereas the results of the previous section prove beyond doubt that an eddy viscosity model cannot correctly predict the perturbation produced by an external volume force, the connection between this result and flow past a wavy bottom relies on the approximate equivalence established in eq.(14). In a later development [34] we overcame this double passage by developing a new computer code, based on the immersed-boundary method, that can handle a wavy bottom directly without any linearization. The new code uses second-order finite differences in all directions, with a stretched coordinate along the wall-normal, $z$ direction only; it has built-in parallelization and uses an explicit time advancement for all but the pressurecorrection equation. Our immersed-boundary implementation uses internal (to the fluid) points only; it is continuous with respect to boundary crossing and stable in iteration at all distances from the boundary. The same discretization was adopted and proved its worth in a previous Stokes-flow application 
[35]. The most delicate part of the procedure turned out to be evaluating the wall shear stress. A commonly adopted solution of summing virtual forces is not sufficient: the contribution of pressure gradient over fractional cell boundaries is of the same order as the effect under investigation and had to be explicitly included in the calculation. Just as in the previous work of [28], the amplitude $\varepsilon$ of the wall oscillation has to strike a compromise between linearity and statistical fluctuation error of time averaging, which had to be found by trial and error. For this purpose a new algorithm was developed [36] in order to estimate the expected error of the time average.
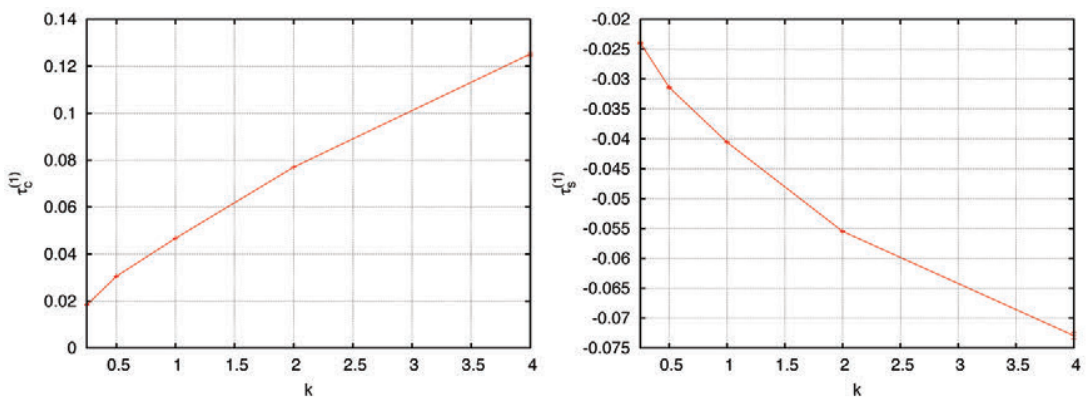

Fig. 10. Turbulent channel: in-phase shear stress (left) and quadrature shear stress (right). $R e=1450(\operatorname{Re}=100)$; computational box up to $8 \pi$.

Our first results, shown in Fig. 10 were generally consistent with the eddy-viscosity finding of a negative quadrature component and seemed to disprove Russo \& Luchini's [28] findings. Nevertheless, at closer inspection, one could notice that the curve of the in-phase component was far from becoming flat at its lefthand side, as it should have been were the wavenumber range wide enough to attain the long-wavelength limit. It looked necessary to attain smaller wavenumber $k$. The smallest achievable wavenumber in a periodic-box DNS is determined by the length of the periodic box, therefore the only way to attain smaller wavenumbers was to increase the computational box and with it the number of discretization points (for a fixed discretization stepsize). After doing so in incremental steps up to a computational box $256 \pi$ long (which required a $12288 \times 160 \times 64$ mesh), we could draw the totally unexpected plot in Fig. 11 here. This was the main result of [34].

As can be seen from Fig. 11, the in-phase component of shear 
stress non-monotonically attains its limiting zero- $k$ value (which can be calculated theoretically and equals the perturbation of the mean stress displayed in green). It also exhibits what looks like a corner singularity, where incidentally its value is nearly exactly zero, at an intermediate wavenumber. This shape hints at the presence of a resonance, although a resonance of this kind is theoretically unknown. At the same time the quadrature components overshoots zero, and in approaching $k=0$ (the long-wave limit), it is indeed positive in accordance with the results of Russo \& Luchini [28].
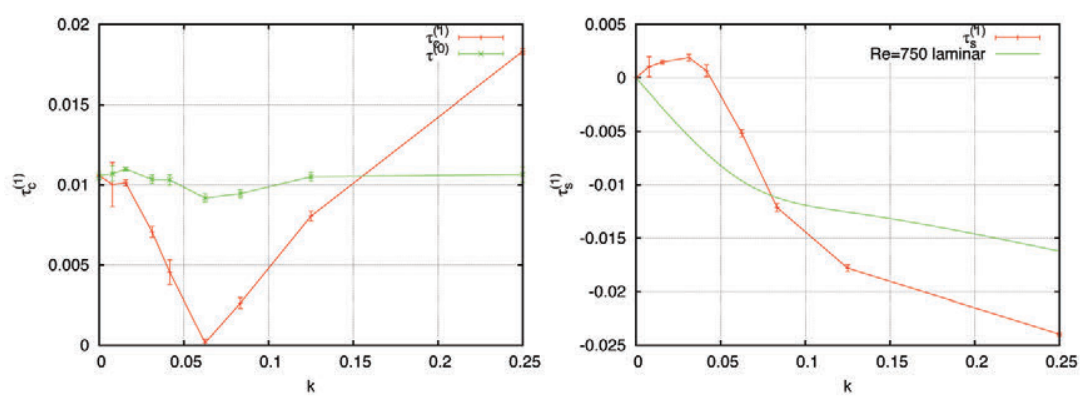

Fig. 11. Turbulent channel: in-phase shear stress (left) and quadrature shear stress (right) for small k. $R e=1450$ ( $R e=100)$; computational box up to $256 \pi$.

\subsection{Effect of Reynolds number}

The initial calculations displayed in Figs. 10-11 were performed at the smallest Reynolds number where turbulence can be sustained for obvious reasons of computational cost; a doubt thus remained that the observed pecularities might be specific to this case. Computer runs at larger Reynolds number, however, essentially confirmed the previous findings. Fig. 12 shows plots of the in-phase and quadrature components of shear stress for three different Reynolds numbers, and Fig. 13 the same data again, but with the horizontal axis normalized in wall units. Although the corner singularity only appears to be present at the smallest Reynolds number (with the fascinating suggestion that a mathematical singularity may actually be present right at transition, and then become smoothed with increasing Reynolds number), the general behaviour of these curves and in particular the overshoot of the quadrature component are confirmed to be generic features. 

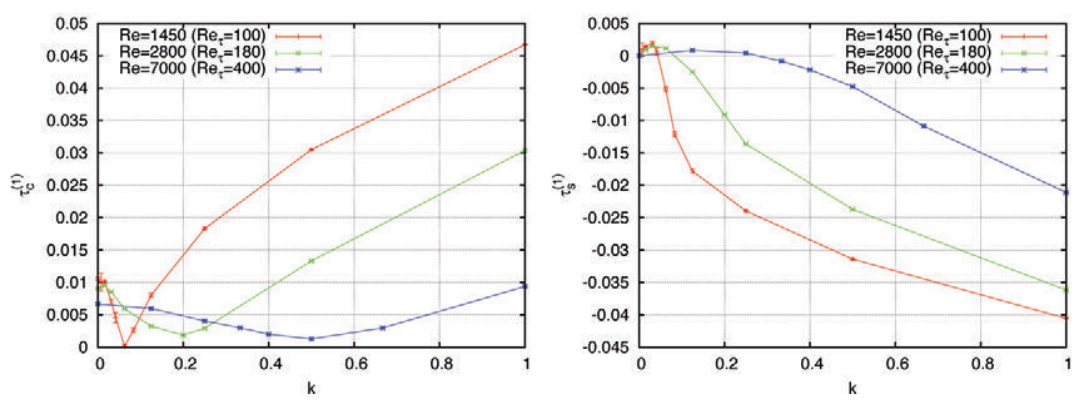

Fig. 12. In-phase (left) and quadrature (right) shear stress perturbations at higher Reynolds numbers.
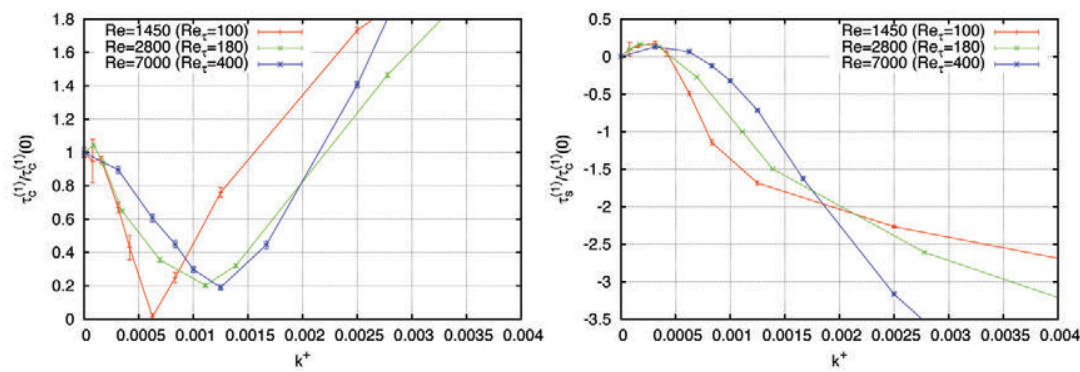

Fig. 13. In-phase (left) and quadrature (right) shear stress perturbations at higher Reynolds numbers versus wavenumber in wall units.

\section{CONCLUSIONS}

In this overview of results obtained through several years, and published in several papers, we have shown that the linear response of turbulent flow to a wavy bottom exhibits a (possibly singular) change of behaviour, and sign, at very large wavelength $\left(\lambda \approx 32 \pi\right.$ when $\operatorname{Re}_{\tau}=100$, or $\lambda^{+} \approx$ 5000 at higher Reynolds numbers). This could only be seen through DNS by adopting a much longer computational box than the usual size of $4 \pi$ generally deemed sufficient for most purposes. No experiment, to our knowledge, has been conducted at such large wavelength, although with hindsight some confirmation can be found [37] in the old experiments of Abrams and Hanratty [38]. In striking contrast to laminar flow (and to all turbulence models), the long-wave quadrature shear stress is positive, just as predicted by the equivalent-force simulations of Russo \& Luchini [28]. 
As far as the implications for shear stress over a dune are concerned, we see that there always is a range of wavenumbers where the quadrature component of shear stress is negative, in agreement with the observation that dunes do unstably grow in practice, but this is not the long-wave limit. Contrary to what happens in laminar flow, in turbulent flow the long-wave limit of quadrature shear stress is positive, just as predicted by the volume-force analogy of [28], but this limit is only achieved at really long wavenumbers. It follows that the long-wave Saint-Venant equations should be applied with great caution to turbulent flow, and in particular not using eddy-viscosity models which fail to predict the correct behaviour. For laminar flow, on the other hand, and for stability calculations of the same, the consistent Saint-Venant equations derived in [1] provide an appropriate tool.

\section{REFERENCES}

[1] Luchini, P. \& Charru, F. (2010), Consistent section-averaged equations of quasione-dimensional laminar flow, J. Fluid Mech. 656, 337-341.

[2] Pedlosky, J. (2003), Waves in the Ocean and Atmosphere, Springer-Verlag.

[3] Whitham, G.B. (1974), Linear and Nonlinear Waves, John Wiley \& Sons, New York.

[4] Hopfinger, E.J. (1983), Snow Avalanche Motion and Related Phenomena, Annu. Rev. Fluid Mech. 15, 47-76.

[5] Aradian, A., Raphael, É. \& de Gennes, P.G. (2002), Surface flows of granular materials: a short introduction to some recent models, C.R. Physique 3, 187-196.

[6] Forterre, Y. and Pouliquen, O. (2008), Flows of Dense Granular Media, Annu. Rev. Fluid Mech. 40, 1-24.

[7] Lin, P.Y. \& Hanratty, T.J. (1984), Prediction of the initiation of slugs with linear stability theory, Intl J. Multiphase Flow 12, 79-98.

[8] Fabre, J. \& Liné, A. (1992), Modeling of Two-Phase Slug Flow, Annu. Rev. Fluid Mech. (24), 21-46.

[9] Jeffreys, H. (1925), The flow of water in an inclined channel of rectangular cross-section, Philos. Mag., Ser. 6 49, 793-807.

[10] Miles, J.W. (1957), On the generation of surface waves by shear flows, J. Fluid Mech. 3, 185-204.

[11] Gradowczyk, M.H. (1968), Wave propagation and boundary instability in erodible-bed channels, J. Fluid Mech. 33, 93-112.

[12] Charru, F. \& Hinch, E.J. (2000), Phase diagram of interfacial instabilities in a two-layer Couette flow and mechanism of the long-wave instability, J. Fluid Mech. 414, 195-233.

[13] Richards, K.J. (1980), The formation of ripples and dunes on an erodible bed, J. Fluid Mech. 99, 597-618. 
[14] Hanratty, T.J. (1983) Interfacial instabilities caused by air flow over a thin liquid layer, in Waves on Fluid Interfaces, Academic press, pp. 221-259.

[15] Hulscher, S.J.M.H., de Swart, H.E., \& de Vriend, H.J. (1993), The generation of offshore tidal sand banks and sand waves, Continental shelf research 13, 1183-1204.

[16] Blondeaux, P. \& Seminara, G. (1985) A unified bar-bend theory of river meanders, J. Fluid Mech. 157, 449-470.

[17] Benjamin, T.B. (1959), Shearing flow over a wavy boundary, J. Fluid Mech. 6, 161-205.

[18] Luchini, P. \& Charru, F. (2010), The phase lead of shear stress in shallow-water flow over a perturbed bottom, J. Fluid Mech. 665, 516-539.

[19] Yih, C.S. (1963) Stability of liquid flow down an inclined plane, Phys. Fluids 6, 321-334.

[20] Bagnold, R.A. (1941) The Physics of Blown Sand and Desert Dunes, Methuen, London.

[21] Charru, F., Andreotti, B. \& Claudin, P. (2013) Sand Ripples and Dunes, Annu. Rev. Fluid Mech. 45, 469-493.

[22] Benjamin, T.B. (1957) Wave formation in laminar flow down an inclined plane, J. Fluid Mech. 2, 554-574.

[23] Jackson, P.S. \& Hunt, J.C.R. (1975), Turbulent wind flow over a low hill, Q. J. R. Meteorol. Soc. 101, 929-955.

[24] Belcher, S.E. \& Hunt, J.C.R. (1998), Turbulent flow over hills and waves, Annu. Rev. Fluid Mech. 30, 507-538.

[25] Hinze J.O. (1975), Turbulence, McGraw Hill.

[26] Nezu, I. \& Rodi, W. (1986), Open-channel flow measurements with a laser doppler anemometer, J. Hydr. Engrg. 112, 335-355.

[27] Jimenez, J., Uhlmann, M., Pinelli, A. \& Kawahara, G. (2001), Turbulent shear flow over active and passive porous surfaces J. Fluid Mech. 442, 89-117.

[28] Russo, S. \& Luchini, P. (2016) The linear response of turbulent flow to a volume force: comparison between eddy-viscosity model and DNS, J. Fluid Mech. 790, 104-127.

[29] Luchini, P. (2008) Acoustic streaming and lower-than-laminar drag in controlled channel flow, Progress in Industrial Mathematics at ECMI 2006, Springer, Dordrecht, 169-177.

[30] Luchini, P. \& Charru, F. (2005) Acoustic streaming past a vibrating wall Phys. Fluids 17, 122106.

[31] Hussain, A.K.M.F. \& Reynolds, W.C. (1970) The mechanics of an organized wave in turbulent shear flow, J. Fluid Mech. 41, 241.

[32] Luchini, P., Quadrio, M. \& Zuccher, S. (2006) The phase-locked mean impulse response of a turbulent channel flow, Phys. Fluids 18, 121702.

[33] Luchini, P. \& Quadrio, M. (2006) A low-cost parallel implementation of direct numerical simulation of wall turbulence J. Comp. Phys. 211, 551-571.

[34] Luchini, P. (2016) Immersed-boundary simulations of turbulent flow past a sinusoidally undulated river bottom Eur. J. Mech. B/Fluids 55, 340-347.

[35] Luchini, P. (2013) Linearized no-slip boundary conditions at a rough surface $J$. Fluid Mech. 737, 349-367. 
[36] Russo, S. \& Luchini, P. (2017) A fast algorithm for the estimation of statistical error in DNS (or experimental) time averages J. Comp. Phys. 347, 328-340.

[37] Luchini, P. (2017) Addendum to "Immersed-boundary simulations of turbulent flow past a sinusoidally undulated river bottom" [Eur. J. Mech. B Fluids 55 (2016) 340-347] Eur. J. Mech. B Fluids 62, 57-58.

[38] Abrams, J. \& Hanratty, T.J. (1985) Relaxation effects observed for turbulent flow over a wavy surface, J. Fluid Mech. 151 443-455. 